\title{
Controllability of a Stochastic Neutral Functional Differential Equation Driven by a fBm
}

\author{
Jingqi Han'1, Litan Yan,2 \\ ${ }^{1}$ College of Information Science and Technology, Donghua University, Shanghai, China \\ ${ }^{2}$ Department of Mathematics, Donghua University, Shanghai, China \\ Email: jingqihan0916@163.com, litan-yan@hotmail.com
}

How to cite this paper: Han, J.Q. and Yan, L.T. (2018) Controllability of a Stochastic Neutral Functional Differential Equation Driven by a $\mathrm{fBm}$. Journal of Applied Mathematics and Physics, 6, 910-924. https://doi.org/10.4236/jamp.2018.64078

Received: March 30, 2018

Accepted: April 24, 2018

Published: April 27, 2018

Copyright ( 92018 by authors and Scientific Research Publishing Inc. This work is licensed under the Creative Commons Attribution International License (CC BY 4.0).

http://creativecommons.org/licenses/by/4.0/

\begin{abstract}
In this paper, we consider a class of Sobolev-type fractional neutral stochastic differential equations driven by fractional Brownian motion with infinite delay in a Hilbert space. When $\alpha>1-H$, by the technique of Sadovskii's fixed point theorem, stochastic calculus and the methods adopted directly from deterministic control problems, we study the approximate controllability of the stochastic system.
\end{abstract}

\section{Keywords}

Fractional Stochastic Neutral Functional Differential Equation, Fractional Brownian Motion, Fractional Calculus, Controllability

\section{Introduction}

As an important part of mathematical control theory, the research on approximate controllability has attracted more and more attention [1] [2] [3]. Approximate controllability means that the system can be steered to a small neighborhood of the final state. In fact, the approximate controllability of systems has been studied by several authors [4] [5]. During the past three decades, the importance of fractional differential equations and their applications are prominent, especially in modeling several complex phenomena such as anomalous diffusion of particles (see, for examples, [6] [7]). In addition, neutral stochastic differential equations with infinite delay have become very useful in the mathematical models of physical and social sciences [8] [9]. So, it is necessarily and significatively to study fractional order neutral differential equations of Sobolev-type ([10] [11] and references therein).

On the other hand, the properties of long/short-range dependence are widely 
used in describing many phenomena in fields like hydrology and geophysics as well as economics and telecommunications. As extension of Brownian motion, fractional Brownian motion ( $\mathrm{fBm}$ ) is a self-similar Gaussian process which has the properties of long/short-range dependence. However, fractional Brownian motion is neither a semimartingale nor a Markov process (except for the case $H=\frac{1}{2}$ when it is a Brownian motion). For this reason, there are a few publications leaning the systems which are driven by this type of noise. We refer [12] [13] and references therein for the details of the theory of stochastic calculus for fractional Brownian motion. In [14], authors consider the approximate controllability of a class of Sobolev-type fractional stochastic equation driven by fractional Brownian motion in a Hilbert space.

Motivated by these results, in this paper, we study the approximate controllability of the Sobolev-type fractional stochastic differential equations of the form

$$
\begin{cases}{ }^{c} D_{t}^{\alpha}\left[L x(t)-G\left(t, x_{t}\right)\right]=A x(t)+f\left(t, x_{t}\right)+B u(t)+\sigma(t) \frac{\mathrm{d}}{\mathrm{d} t} B^{H}(t), & t \in(0, T], \\ x(t)=\phi(t), & t \in(-\infty, 0] .\end{cases}
$$

In the above system, we assume that

- ${ }^{c} D^{\alpha}$ is the Caputo fractional derivative of order $\alpha \in(1-H, 1)$,

- $A, L$ are two linear operators on a Hilbert space $U$,

- $B$ is a bounded linear operator from the Hilbert space $V$ into Hilbert space $U$,

- The time history $x_{t}(\theta)=x(t+\theta), t>0$,

- $u(\cdot)$ is a control function on $L^{2}([0, T], V)$,

- $B^{H}=\left\{B^{H}(t), t \in[0, T]\right\}$ is a cylindrical fractional Brownian motion with Hurst index $H \in\left(\frac{1}{2}, 1\right)$,

- The functions $G$, $f$ and $\sigma$ are Borel functions with some suitable conditions.

The paper is organized as follows. In Section 2, we represent some preliminaries for stochastic integral of fractional Brownian motion in Hilbert space. In Section 3, we obtain the approximate controllability results of the Sobolev-type fractional neutral stochastic system (1.1).

\section{Preliminaries}

In this section, we will introduce some definitions, lemmas and notions which will be used in the next section.

\subsection{Fractional Brownian Motion}

Let $\left(\Omega, \mathscr{F},\left(\mathscr{T}_{t}\right), \mathrm{P}\right)$ be a complete filtered probability space. A fractional Brownian motion (fBm) $\beta^{H}=\left\{\beta^{H}(t), t \in[0, T]\right\}$ with Hurst index $H \in(0,1)$ is a mean zero Gaussian process such that $\beta^{H}(0)=0$ and 


$$
\mathrm{E}\left(\beta^{H}(t) \beta^{H}(s)\right)=\frac{1}{2}\left(s^{2 H}+t^{2 H}-|t-s|^{2 H}\right)
$$

for all $t, s \geq 0$. When $H=1 / 2, \beta^{H}$ coincides with the standard Brownian motion, and when $H \neq \frac{1}{2}$ it is neither a semi-martingale nor a Markov process. The $\mathrm{fBm} \quad \beta^{H}$ admits the following integral representation:

$$
\beta^{H}(t)=\int_{0}^{t} K_{H}(t, s) \mathrm{d} W(t)
$$

for all $t \geq 0$, where $\{W(t), 0 \leq t \leq T\}$ is a standard Brownian motion and the kernel $K_{H}(t, s)$ satisfies

$$
\frac{\partial K_{H}}{\partial t}(t, s)=\kappa_{H}\left(H-\frac{1}{2}\right)\left(\frac{s}{t}\right)^{\frac{1}{2}-H}(t-s)^{H-\frac{3}{2}}
$$

with a normalizing constant $\kappa_{H}>0$ such that $E\left(\beta_{1}^{H}\right)^{2}=1$. Throughout this paper we assume that $\frac{1}{2} \leq H<1$ is arbitrary but fixed.

Let $\mathcal{H}$ be the completion of the linear space $\mathcal{E}$ generated by the indicator functions $1_{[0, t]}, t \in[0, T]$ with respect to the inner product

$$
\left\langle 1_{[0, s]}, 1_{[0, t]}\right\rangle_{\mathcal{H}}=\frac{1}{2}\left(t^{2 H}+s^{2 H}-|t-s|^{2 H}\right) .
$$

The mapping

$$
\mathcal{E} \ni \varphi \rightarrow \beta^{H}(\varphi):=\int_{0}^{T} \varphi(s) \mathrm{d} \beta^{H}(s)
$$

is an isometry from $\mathcal{E}$ to the Gaussian space generated by $\beta^{H}$ and it can be extended to $\mathcal{H}$, which is called the Wiener integral with respect to $\beta^{H}$. Consider the operator $K_{H}^{*}$ from $\mathcal{E}$ to $L^{2}([0, T])$ defined by

$$
\left(K_{H}^{*} \varphi\right)(s)=\int_{s}^{T} \varphi(t) \frac{\partial K_{H}}{\partial t}(t, s) \mathrm{d} t
$$

for $\varphi \in \mathcal{E}$. Then, the operator $K_{H}^{*}$ is an isometry between $\mathcal{E}$ and $L^{2}([0, T])$ which can be also extended to the Hilbert space $\mathcal{H}$.

Lemma 2.1 For every $\varphi \in \mathcal{H}$, we have

$$
\int_{0}^{T} \varphi(s) \mathrm{d} \beta^{H}(s)=\int_{0}^{T}\left(K_{H}^{*} \varphi\right)(s) \mathrm{d} W(s) .
$$

We now recall that the definition of stochastic integral of $\mathrm{fBm}$ in the Hilbert space $V$. Let $\left\{B^{H}(t), 0 \leq t \leq T\right\}$ be a $W$-valued $\mathscr{F}_{t}$-adapted $\mathrm{fBm}$ defined on $\left(\Omega, \mathscr{F},\left(\mathscr{F}_{t}\right), \mathrm{P}\right)$ with the representation of the form

$$
B^{H}(t)=\sum_{n=1}^{\infty} \sqrt{\lambda_{n}} \beta_{n}^{H}(t) e_{n}, \quad t \geq 0,
$$

where $\left\{e_{n}\right\}_{n \in N}$ is a complete orthogonal basis in $W$, and

- $\left\{\beta_{n}^{H}, n=1,2, \cdots\right\}$ is a sequence of independent $\mathrm{fBms}$ with the same Hurst index $H \in\left(\frac{1}{2}, 1\right)$, 
- $\left\{\lambda_{n} ; n \in \mathbb{N}\right\}$ is a bounded sequence of non-negative real numbers such that $Q e_{n}=\lambda_{n} e_{n}$,

- $Q$ is a non-negative self-adjoint trace class operator with finite trace

$$
\operatorname{Tr} Q=\sum_{n=1}^{\infty} \lambda_{n}<+\infty
$$

Let $\varphi:[0, T] \rightarrow L_{2}^{0}(W, U)$ such that

$$
\sum_{n=1}^{\infty}\left\|K_{H}^{*}\left(\varphi Q^{\frac{1}{2}} e_{n}\right)\right\|_{L_{2}^{0}([0, T] ; U)}<\infty
$$

where $L_{2}^{0}(W, U)$ is the space of all Hilbert-Schmidt operators from $Q^{\frac{1}{2}} W$ to $U$ with norm $\|\cdot\|_{L_{2}^{0}(W, U)}$ defined by

$$
\|\xi\|_{L_{2}^{0}(W, U)}=\left\|\xi Q^{\frac{1}{2}}\right\|_{H-S}^{2}=\operatorname{Tr}\left(\xi Q \xi^{*}\right)=\sum_{n=1}^{\infty}\left\|\sqrt{\lambda_{n}} \xi e_{n}\right\|^{2} .
$$

Definition 2.1 Let $\varphi:[0, T] \rightarrow L_{2}^{0}(W, U)$ satisfy (2.1). We define the stochastic integral $\int_{0}^{t} \varphi(s) \mathrm{d} B^{H}(s)$ by

$$
\int_{0}^{t} \varphi(s) \mathrm{d} B^{H}(s):=\sum_{n=1}^{\infty} \int_{0}^{t} \varphi(s) Q^{\frac{1}{2}} e_{n} \mathrm{~d} \beta_{n}^{H}=\sum_{n=1}^{\infty} \int_{0}^{t}\left(K_{H}^{*}\left(\varphi Q^{\frac{1}{2}} e_{n}\right)\right)(s) \mathrm{d} B(s) .
$$

Lemma 2.2 Let $\varphi:[0, T] \rightarrow L_{2}^{0}(W, U)$ satisfy (2.1). Then, for any $a, b \in[0, T]$ with $a<b$ we have

$$
\mathrm{E}\left\|\int_{a}^{b} \varphi(s) \mathrm{d} B^{H}(s)\right\|^{2} \leq c H(2 H-1)(b-a)^{2 H-1} \sum_{n=1}^{\infty} \int_{a}^{b}\left\|\varphi(s) Q^{\frac{1}{2}} e_{n}\right\|^{2} \mathrm{~d} s .
$$

In addition, $\sum_{n=1}^{\infty}\left\|\varphi(s) Q^{\frac{1}{2}} e_{n}\right\|$ is uniformly convergent in $t \in[0, T]$, then, we have

$$
\mathrm{E}\left\|\int_{a}^{b} \varphi(s) \mathrm{d} B^{H}(s)\right\|^{2} \leq c H(2 H-1)(b-a)^{2 H-1} \sum_{n=1}^{\infty} \int_{a}^{b}\|\varphi(s)\|_{L_{2}^{0}(W, U)}^{2} \mathrm{~d} s .
$$

\subsection{Some Assumptions}

In this subsection, we recall that some notions of fractional calculus and give some assumptions for the stochastic system (1.1). Recall that the fractional integral $I^{\alpha}$ of order $\alpha$ for a function $f:[0, \infty) \rightarrow R$ is defined as

$$
I^{\alpha} f(t)=\frac{1}{\Gamma(\alpha)} \int_{0}^{t} \frac{f(s)}{(t-s)^{1-\alpha}} \mathrm{d} s, \quad t>0, \alpha>0,
$$

provided the right side is point-wise defined on $[0, \infty)$, where $\Gamma(\cdot)$ is the gamma function, which is defined by $\Gamma(x):=\int_{0}^{\infty} t^{x-1} \mathrm{e}^{-t} \mathrm{~d} t$. Moreover, the Caputo derivative ${ }^{c} D^{\alpha}$ of order $\alpha$ for a function $f \in \mathbf{C}^{n}([0, \infty))$ is defined as 


$$
{ }^{c} D_{t}^{\alpha} f(t)=\frac{1}{\Gamma(n-\alpha)} \int_{0}^{t} \frac{f^{(n)}(s)}{(t-s)^{1+\alpha-n}} \mathrm{~d} s=I^{n-\alpha} f^{(n)}(t), \quad t>0, n-1<\alpha<n .
$$

If $f$ is an abstract function with values in $U$, then the integrals appearing in the above definitions are taken in Bochner's sense.

To study the stochastic system (1.1), we need some assumptions. Throughout this paper we assume that $U, V, W$ is three real separable Hilbert spaces with inner products $\langle\cdot, \cdot\rangle_{U},\langle\cdot, \cdot\rangle_{V}$ and $\langle\cdot, \cdot\rangle_{W}$, respectively. We first give some conditions about the three operators $L, A, B$ as follows:

(A1) $A$ and $L$ are two linear operators on $U$ such that $D(A) \subset U, D(L) \subset U$, and $A$ is closed,

(A2) $D(L) \subset D(A)$ and $L$ is bijective,

(A3) $L^{-1}: U \rightarrow D(U)$ is compact,

(A4) $B$ is a bounded linear operator from $V$ into $U$.

From the above assumptions $(\mathbb{A} 1)-(\mathbb{A} 3)$ and the closed graph theorem it follows that the linear operator $A L^{-1}: U \rightarrow U$ is bounded, and $A L^{-1}$ generates a semigroup $\{S(t), t>0\}$ in $U$. Denote $M=\max _{t>0}\|S(t)\|,\|L\|=M_{1}$ and $\left\|L^{-1}\right\|=\widetilde{M_{1}}$.

For $x \in U$, we define two families $\left\{\mathscr{T}_{L}(t), t \geq 0\right\}$ and $\left\{\mathscr{C}_{L}(t), t \geq 0\right\}$ of operators by

$$
\mathscr{T}_{L}(t) x:=\int_{0}^{\infty} L^{-1} \xi_{\alpha}(\theta) S\left(t^{\alpha} \theta\right) x \mathrm{~d} \theta
$$

and

$$
\mathscr{C}_{L}(t) x:=\alpha \int_{0}^{\infty} L^{-1} \theta \xi_{\alpha}(\theta) S\left(t^{\alpha} \theta\right) x \mathrm{~d} \theta
$$

where

$$
\theta \mapsto \xi_{\alpha}(\theta)=\frac{1}{\pi \alpha} \sum_{n=1}^{\infty}(-\theta)^{n-1} \frac{1}{n !} \Gamma(n \alpha+1) \sin (n \pi \alpha)
$$

is a probability density function defined on $(0, \infty)$.

Lemma 2.3 Feckan, M. et al. [15] The operators $\mathscr{T}_{L}(t)$ and $\mathscr{S}_{L}(t)$ have the following properties:

- For every $t \geq 0, \mathscr{T}_{L}(t)$ and $\mathscr{S}_{L}(t)$ are linear and bounded, and moreover for every $x \in U$

$$
\begin{aligned}
& \left\|\mathscr{\sigma}_{L}(t) x\right\| \leq M \widetilde{M}_{1}\|x\|, \\
& \left\|\mathscr{C}_{L}(t) x\right\| \leq \frac{M \widetilde{M}_{1}}{\Gamma(\alpha)}\|x\| .
\end{aligned}
$$

- $\mathscr{T}_{L}(t)$ and $\mathscr{S}_{L}(t)$ are strong continuous and compact.

We now introduce the abstract phase space. For a continuous function $h:(-\infty, 0] \rightarrow(0, \infty)$ satisfying

$$
l:=\int_{-\infty}^{0} h(t) \mathrm{d} t<\infty,
$$

we define a phase space 


$$
\begin{aligned}
\mathscr{P}=\{ & \phi:(-\infty, 0] \rightarrow U, \text { for any } a>0,\left(\mathrm{E}\|\phi(\theta)\|^{2}\right)^{1 / 2} \text { is bounded } \\
& \text { and measurable functions on }[-a, 0] \text { with } \phi(0)=0 \\
& \text { and } \left.\int_{-\infty}^{0} h(s) \sup _{s \leq \theta \leq 0}\left(\mathrm{E}\|\phi(\theta)\|^{2}\right)^{1 / 2} \mathrm{~d} s<\infty\right\} .
\end{aligned}
$$

Clearly, $\left(\mathscr{P}_{h},\|\cdot\|_{\mathscr{h}_{h}}\right)$ is a Banach space if $\mathscr{P}_{h}$ is endowed with the norm (see, Cui and Yan [16])

$$
\|\phi\|_{h}=\int_{-\infty}^{0} h(s) \sup _{s \leq \theta \leq 0}\left(\mathrm{E}\|\phi(\theta)\|^{2}\right)^{1 / 2} \mathrm{~d} s
$$

for $\phi \in \mathscr{P}$.

We present the definition of mild solutions of (1.1).

Definition 2.2 An $U$-valued stochastic process $\{x(t), t \in[-\infty, T]\}$ is a mild solution of (1.1) if the next conditions hold:

i) $x(t)$ is measurable and $\mathscr{F}_{t}$-adapted, and $x_{t}$ is $\mathscr{F}_{h}$-valued,

ii) $x(t)$ is continuous on $[0, T]$ and the function

$(t-s)^{\alpha-1} L^{-1} A \mathscr{S}_{L}(t-s) G\left(t, x_{s}\right)$ is integrable for each $s \in[0, t]$ such that $x(t)$ satisfies the equation

$$
\begin{aligned}
x(t)= & \mathscr{T}_{L}(t)(L \phi(0)-G(0, \phi))+L^{-1} G\left(t, x_{t}\right) \\
& +\int_{0}^{t}(t-s)^{\alpha-1} L^{-1} A \mathscr{O}_{L}(t-s) G\left(t, x_{s}\right) \mathrm{d} s \\
& +\int_{0}^{t}(t-s)^{\alpha-1} \mathscr{T}_{L}(t-s)\left[f\left(s, x_{s}\right)+B u(s)\right] \mathrm{d} s \\
& +\int_{0}^{t}(t-s)^{\alpha-1} \mathscr{S}_{L}(t-s) \sigma(s) \mathrm{d} B^{H}(s),
\end{aligned}
$$

iii) $x(t)=\phi(t)$ on $(-\infty, 0]$ such that $\|\phi\|_{S_{h}}^{2}<\infty$.

Finally, in order to prove our main statement, we need some conditions as follows.

(B1) Let the function $f:[0, T] \times, P_{h} \rightarrow U$ is continuous and there exist some constants $N_{f}>0, k_{f}>0$ such that for $t \in[0, T]$ and $\xi, \eta \in \mathscr{P}_{h}$

$$
\mathrm{E}\|f(t, \xi)-f(t, \eta)\|^{2} \leq N_{f}\|\xi-\eta\|_{h_{h}}^{2}
$$

for all $t \in[0, T]$ and $k_{f}=\sup _{t \in[0, T]}\|f(t, 0)\|^{2}$.

(B2) For the complete orthogonal basis $\left\{e_{n}\right\}_{n \in \mathbb{N}}$ in $W$, the function $\sigma:[0, T] \rightarrow L_{2}^{0}(W, U)$ satisfy

$$
\sum_{n=1}^{\infty}\left\|\sigma Q^{\frac{1}{2}} e_{n}\right\|_{L^{2}([0, T], U)}<\infty
$$

and $\sum_{n=1}^{\infty}\left\|\sigma(t) Q^{\frac{1}{2}} e_{n}\right\|$ is uniformly convergent in $t \in[0, T]$. In addition, there exist some $t_{0}$ and $\delta>0$ such that

$$
\int_{0}^{t_{0}} \int_{0}^{t_{0}} r^{-\delta} s^{-\delta}\|\sigma(r)\|_{L_{2}^{0}(W, U)}\|\sigma(s)\|_{L_{2}^{0}(W, U)} \mathrm{d} r \mathrm{~d} s<\infty .
$$


(B3) Let the function $G:[0, T] \times \mathscr{S}_{\mathrm{h}} \rightarrow U$ is continuous and satisfies:

(a) there exist some constants $N_{G}>0, k_{G}>0$ for $t, s \in[0, T]$ and $\xi, \eta \in \mathscr{P}_{\mathrm{h}}$ such that the function $A G$ satisfies the Lipschitz condition

$$
\mathrm{E}\|A G(t, \xi)-A G(t, \eta)\|^{2} \leq N_{G}\|\xi-\eta\|_{\text {h }_{h}}^{2}
$$

for all $t \in[0, T]$ and $k_{g}=\sup _{t \in[0, T]}\|A G(t, 0)\|^{2}$.

(b) there exist constants $\widetilde{N_{G}}>0, \widetilde{k_{g}}>0$ such that

$$
\mathrm{E}\|G(t, \xi)-G(t, \eta)\|^{2} \leq \widetilde{N_{G}}\|\xi-\eta\|_{\text {, }_{h}}^{2}
$$

for all $t \in[0, T], \quad \xi, \eta \in \mathscr{P}$ and $\widetilde{k_{g}}=\sup _{t \in[0, T]}\|G(t, 0)\|^{2}$.

(B4) There is a constant $\mathscr{K}>0$ such that $\frac{\mathcal{K}_{1} \mathscr{K}}{\mathcal{K}_{2}}>1$, where

$$
\begin{aligned}
& \mathcal{K}_{1}=1-24 l^{2}\left(\frac{M \widetilde{M}_{1} T^{\alpha}}{\alpha \Gamma(\alpha)}\right)^{2} N_{f}\left(1+4\left(\frac{M^{2} \widetilde{M}_{1}^{2} M_{B} T^{\alpha}}{\alpha \lambda \Gamma(\alpha)^{2}}\right)^{2}\right)>0, \\
& \mathcal{K}_{2}=4 l^{2}\left(M M_{1} \widetilde{M}_{1}\right)^{2} \mathrm{E}\left\|\phi_{0}\right\|^{2}\left[1+12\left(\frac{M^{2} \widetilde{M}_{1}^{2} M_{B}^{2} T^{\alpha}}{\alpha \lambda \Gamma(\alpha)^{2}}\right)^{2}\right]+4\|\phi\|_{S_{h}}^{2} \\
& +12 l^{2}\left(\frac{M \widetilde{M}_{1}}{\Gamma(\alpha)}\right)^{2}\left[1+4\left(\frac{M^{2} \widetilde{M}_{1}^{2} M_{B}^{2} T^{\alpha}}{\alpha \lambda \Gamma(\alpha)^{2}}\right)\right] \times\left(\frac{2 k_{f} T^{2 \alpha}}{\alpha^{2}}+c H(2 H-1) N_{\sigma}\right) \\
& +96 l^{2}\left(\frac{M^{2} \widetilde{M}_{1}^{2} M_{B}^{2} T^{\alpha}}{\alpha \lambda \Gamma(\alpha)^{2}}\right)^{2}\left(\mathrm{E}\left\|z_{T}\right\|^{2}+c H(2 H-1) T^{2 H-1} \int_{0}^{T}\|\hat{\varphi}(s)\|_{L_{2}^{0}(W, U)}^{2} \mathrm{~d} s\right), \\
& N_{\sigma}:=\sup _{t \in[0, T]} \int_{0}^{t} \int_{0}^{t}(t-s)^{\alpha-1}(t-r)^{\alpha-1}|s-r|^{2 H-2}\|\sigma(s)\|_{L_{2}^{0}(W, U)}\|\sigma(r)\|_{L_{2}^{0}(W, U)} \mathrm{d} s \mathrm{~d} r<\infty, \\
& \text { and } M_{B}=\|B\| .
\end{aligned}
$$

\section{Main Results}

In this section, we will show the approximate controllability of the stochastic system (1.1). We need to establish the existence of the solution for the stochastic control system and to show that the corresponding linear part is approximate controllability.

Definition 3.1 The system (1.1) is called to be approximately controllable on $[0, T]$ if

$$
\overline{\Re(T)}=U
$$

with $\mathfrak{R}(t)=\left\{x(t)=x(t, u): u \in L^{2}([0, T], V)\right\}$.

Consider the corresponding linear fractional deterministic control system to $(1.1)$ 


$$
\left\{\begin{array}{l}
{ }^{c} D_{t}^{\alpha}[L x(t)]=A x(t)+B u(t), \quad t \in[0, T] \\
x(0)=\phi(0),
\end{array}\right.
$$

and define the relevant operators

$$
\Gamma_{0}^{T}=\int_{0}^{T}(T-s)^{\alpha-1} \mathscr{S}_{L}(T-s) B B^{*} \mathscr{T}_{L}^{*}(T-s) \mathrm{d} s
$$

and

$$
R\left(\alpha, \Gamma_{0}^{T}\right)=\left(\alpha I+\Gamma_{0}^{T}\right)^{-1},
$$

where $B^{*}$ and $\mathscr{S}_{L}^{*}(T-s)$ denote the adjoint operators of $B$ and $\mathscr{S}_{L}(T-s)$, respectively. It is clear that the operator $\Gamma_{0}^{T}$ is a linear bounded operator. The fact that the linear Sobolev-type fractional control system (3.2) is approximately controllable on $[0, T]$ is equivalent to the next hypothesis (see, for example, Mahmudov and Denker [17]):

- $\alpha R\left(\alpha, \Gamma_{0}^{T}\right) \rightarrow 0$ in the strong operator topology, as $\alpha \rightarrow 0^{+}$.

Lemma 3.1 (Guendouzi and Idrissi [18]) For any $z_{T} \in L^{2}(\Omega ; U)$, there exists $\hat{\varphi}(t) \in L^{2}\left(\Omega, L^{2}\left(0, T ; L_{2}^{0}\right)\right)$ such that

$$
z_{T}=E z_{T}+\int_{0}^{T} \hat{\varphi}(s) \mathrm{d} B^{H}(s) .
$$

For any $\lambda>0$ and $z_{T} \in L^{2}(\Omega ; U)$, we now define the control function $u^{\lambda}$ as follows.

$$
\begin{aligned}
u^{\lambda}(t)= & B^{*} \mathscr{S}_{L}^{*}(T-t)\left(\lambda I+\Gamma_{0}^{T}\right)^{-1}\left[-\mathscr{T}_{L}(T)(L \phi(0)-G(0, \phi))-L^{-1} G\left(T, x_{T}\right)\right] \\
& +B^{*} \mathscr{L}_{L}^{*}(T-t)\left(\lambda I+\Gamma_{0}^{T}\right)^{-1}\left[\mathrm{E} z_{T}+\int_{0}^{T} \hat{\varphi}(s) \mathrm{d} B^{H}(s)\right] \\
& -B^{*} \mathscr{L}_{L}^{*}(T-t) \int_{0}^{t}\left(\lambda I+\Gamma_{s}^{T}\right)^{-1}(T-s)^{\alpha-1} A \mathscr{S}_{L}(T-s) G\left(s, x_{s}\right) \mathrm{d} s \\
& -B^{*} \mathscr{O}_{L}^{*}(T-t) \int_{0}^{t}\left(\lambda I+\Gamma_{s}^{T}\right)^{-1}(T-s)^{\alpha-1} \mathscr{S}_{L}(T-s) f\left(s, x_{s}\right) \mathrm{d} s \\
& -B^{*} \mathscr{L}_{L}^{*}(T-t) \int_{0}^{t}\left(\lambda I+\Gamma_{s}^{T}\right)^{-1}(T-s)^{\alpha-1} \mathscr{S}_{L}(T-s) \sigma(s) \mathrm{d} B^{H}(s) .
\end{aligned}
$$

Theorem 3.1 (Daher [19]) Let $\Phi$ be a condensing operator on a Banach space $X$, that is, $\Phi$ is continuous and takes bounded sets into bounded sets, and $\mu(\Phi(B)) \leq \mu(B)$ for every bounded set $B$ of $X$ with $\mu(B)>0$. If $\Phi(N) \subset N$ for a convex, closed and bounded set $N$ of $X$, then $\Phi$ has a fixed point in $X$ (where $\mu(\cdot)$ denotes Kuratowski's measure of noncompactness).

Define the space

$$
\mathscr{P}_{a}=\left\{x: x \in C((-\infty, T], U) \text { with } x_{0}=\phi \in \mathscr{P _ { h }}\right\}
$$

and let $\|\cdot\|_{a}$ be a seminorm defined by

$$
\|x\|_{a}=\|\phi\|_{\mathscr{h}_{h}}+\sup _{t \in[0, T]}\left(\mathrm{E}\|x(s)\|^{2}\right)^{\frac{1}{2}}, x \in \mathscr{P},
$$

where $C((-\infty, T], U)$ denotes the space of all continuous $U$-valued stochastic process $\{\xi(t), t \in(-\infty, T]\}$.

Lemma 3.2 ( $\mathrm{Li}$ and Liu [20]) Assume that $x \in \mathscr{P} a$, then for all $t \in[0, T], x_{t} \in P_{h}$. Moreover, 


$$
l\left(\mathrm{E}\|x(t)\|^{2}\right)^{1 / 2} \leq l \sup _{s \in[0, t]}\left(\mathrm{E}\|x(s)\|^{2}\right)^{1 / 2}+\left\|x_{0}\right\|_{\text {多 }},
$$

where $l=\int_{-\infty}^{0} h(s) \mathrm{d} s$ is given in Section 2 .

Theorem 3.2 Assume the conditions (B1)-(B4) hold, then for each $\lambda>0$ there exists a mild solution of $(1.1)$ on $(-\infty, T]$, provided that

$4 l^{2}\left(\widetilde{M}_{1}^{2} \widetilde{N}_{G}+\left(\frac{\widetilde{M}_{1}^{2} M T^{\alpha}}{\alpha \Gamma(\alpha)}\right)^{2} N_{G}\right)<1$.

Proof. Define the operator $\Phi: \mathscr{P}_{a} \rightarrow \mathscr{P}_{a}$ by

$$
\Phi x(t)= \begin{cases}\phi(t), & t \in(-\infty, 0], \\ \mathscr{T}_{L}(t)(L \phi(0)-G(0, \phi))+L^{-1} G\left(t, x_{t}\right) & \\ +\int_{0}^{t}(t-s)^{\alpha-1} L^{-1} A \mathscr{S}_{L}(t-s) G\left(s, x_{s}\right) \mathrm{d} s & \\ +\int_{0}^{t}(t-s)^{\alpha-1} \mathscr{S}_{L}(t-s)\left[f\left(s, x_{s}\right)+B u^{\lambda}(s)\right] \mathrm{d} s & \\ +\int_{0}^{t}(t-s)^{\alpha-1} \mathscr{S}_{L}(t-s) \sigma(s) \mathrm{d} B^{H}(s), & t \in(0, T],\end{cases}
$$

for $x \in \mathscr{P}_{a}$.

We will show that $\Phi$ has a fixed point which is a mild solution for system (1.1). For $\phi \in \mathscr{Y}$, define

$$
\tilde{\phi}(t)= \begin{cases}\phi(t), & t \in(-\infty, 0], \\ \mathscr{F}_{L}(t)(L \phi(0)), & t \in(0, T] .\end{cases}
$$

Then, $\tilde{\phi}(t) \in \mathscr{P}_{a}$. Let $x(t)=\tilde{\phi}(t)+y(t), t \in(-\infty, T]$. It is easy to check that $x(t)$ satisfies (1.1) if and only if $y_{0}=0$ and

$$
\begin{aligned}
y(t)= & -\mathscr{T}_{L}(t) G(0, \phi)+L^{-1} G\left(t, y_{t}+\tilde{\phi}_{t}\right) \\
& +\int_{0}^{t}(t-s)^{\alpha-1} L^{-1} A \mathscr{O}_{L}(t-s) G\left(s, y_{s}+\tilde{\phi}_{s}\right) \mathrm{d} s \\
& +\int_{0}^{t}(t-s)^{\alpha-1} \mathscr{T}_{L}(t-s)\left[f\left(s, y_{s}+\tilde{\phi}_{s}\right)+B u^{\lambda}(s)\right] \mathrm{d} s \\
& +\int_{0}^{t}(t-s)^{\alpha-1} \mathscr{S}_{L}(t-s) \sigma(s) \mathrm{d} B^{H}(s) .
\end{aligned}
$$

Denote $\mathscr{P}_{b}=\left\{y \in \mathscr{P}_{a}, y_{0}=0 \in \mathscr{P}_{\hat{\partial}}\right\}$ and let $\|\cdot\|_{b}$ be the seminorm in $\mathscr{P}_{b}$, defined by

$$
\|y\|_{b}=\left\|y_{0}\right\|_{h}+\sup _{s \in[0, T]}\left(\mathrm{E}\|y(s)\|^{2}\right)^{1 / 2}=\sup _{s \in[0, T]}\left(\mathrm{E}\|y(s)\|^{2}\right)^{1 / 2} .
$$

For $r \geq 0$ we set $B_{r}=\left\{y \in \mathscr{b},\|y\|_{b}^{2} \leq r\right\}$. Then, $B_{r}$ is a bounded closed convex set in $\mathscr{F}_{b}$ for each $r$. According to Lemma 3.2, we get

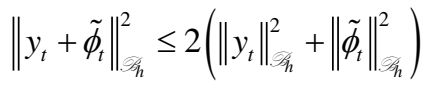

$$
\begin{aligned}
& \leq 4\left(l^{2} \sup _{s \in[0, t]} \mathrm{E}\|y(s)\|^{2}+\left\|y_{0}\right\|_{\boldsymbol{h}_{\boldsymbol{h}}}^{2}+l^{2} \sup _{s \in[0, t]} \mathrm{E}\|\tilde{\phi}(s)\|^{2}+\left\|\tilde{\phi}_{0}\right\|_{\mathbf{h}_{\boldsymbol{h}}}^{2}\right) \\
& \leq 4\left(l^{2} r+l^{2} M^{2} M_{1}^{2} \widetilde{M}_{1}^{2} \mathrm{E}\|\phi(0)\|^{2}+\|\phi\|_{\mathbf{s}_{h}}^{2}\right) \equiv r^{\prime}
\end{aligned}
$$


for $y \in B_{r}$. Define the mapping $\Psi: \mathscr{P}_{b} \rightarrow \mathscr{P}_{b}$ by

$$
\begin{aligned}
\Psi y(t)= & -\mathscr{T}_{L}(t) G(0, \phi)+L^{-1} G\left(t, y_{t}+\tilde{\phi}_{t}\right) \\
& +\int_{0}^{t}(t-s)^{\alpha-1} L^{-1} A \mathscr{C}_{L}(t-s) G\left(s, y_{s}+\tilde{\phi}_{s}\right) \mathrm{d} s \\
& +\int_{0}^{t}(t-s)^{\alpha-1} \mathscr{S}_{L}(t-s)\left[f\left(s, \tilde{\phi}_{s}+y_{s}\right)+B u^{\lambda}(s)\right] \mathrm{d} s \\
& +\int_{0}^{t}(t-s)^{\alpha-1} \mathscr{S}_{L}(t-s) \sigma(s) \mathrm{d} B^{H}(s)
\end{aligned}
$$

for $t \in[0, T]$. It is evident that the operator $\Phi$ has a fixed point if and only if the operator $\Psi$ has a fixed point. Now, we divide $\Psi$ into $\Psi=\Psi_{1}+\Psi_{2}$, where

$$
\begin{aligned}
\Psi_{1} y(t)= & -\mathscr{T}_{L}(t) G(0, \phi)+L^{-1} G\left(t, y_{t}+\tilde{\phi}_{t}\right) \\
& +\int_{0}^{t}(t-s)^{\alpha-1} L^{-1} A \mathscr{L}_{L}(t-s) G\left(s, y_{s}+\tilde{\phi}_{s}\right) \mathrm{d} s, \\
\Psi_{2} y(t)= & \int_{0}^{t}(t-s)^{\alpha-1} \mathscr{S}_{L}(t-s)\left[f\left(s, \tilde{\phi}_{s}+y_{s}\right)+B u^{\lambda}(s)\right] \mathrm{d} s \\
& +\int_{0}^{t}(t-s)^{\alpha-1} \mathscr{S}_{L}(t-s) \sigma(s) \mathrm{d} B^{H}(s) .
\end{aligned}
$$

Now, we need to prove the operator $\Psi_{1}$ is a contraction map and $\Psi_{2}$ is compact.

Step I. $\Psi_{1}$ is a contraction map. For $y, \bar{y} \in \mathscr{P}_{b}$, we have

$$
\begin{aligned}
& \mathrm{E}\left\|\Psi_{1} y(t)-\Psi_{1} \bar{y}(t)\right\|^{2} \\
& \leq 2 \mathrm{E}\left\|L^{-1} G\left(t, y_{t}+\tilde{\phi}_{t}\right)-L^{-1} G\left(t, \bar{y}_{t}+\tilde{\phi}_{t}\right)\right\|^{2} \\
& +2 \mathrm{E}\left\|\int_{0}^{t}(t-s)^{\alpha-1} L^{-1} A \mathscr{S}_{L}(t-s)\left[G\left(s, y_{s}+\tilde{\phi}_{s}\right)-G\left(s, \bar{y}_{s}+\tilde{\phi}_{s}\right)\right] \mathrm{d} s\right\|^{2} \\
& \leq 2 \widetilde{M}_{1}^{2} \widetilde{N}_{G}\left\|y_{t}-\bar{y}_{t}\right\|_{\mathscr{h}_{h}}^{2}+\left(\frac{\widetilde{M}_{1}^{2} M T^{\alpha}}{\alpha \Gamma(\alpha)}\right)^{2} N_{G}\left\|y_{t}-\bar{y}_{t}\right\|_{\cdot_{h}}^{2} \\
& \leq 4 l^{2}\left(\widetilde{M}_{1}^{2} \widetilde{N}_{G}+\left(\frac{\widetilde{M}_{1}^{2} M T^{\alpha}}{\alpha \Gamma(\alpha)}\right)^{2} N_{G}\right) \sup _{t \in[0, T]}\left\|y_{t}-\bar{y}_{t}\right\|^{2} .
\end{aligned}
$$

It follows that $\Psi_{1}$ is a contraction map with the assumption $4 l^{2}\left(\widetilde{M}_{1}^{2} \widetilde{N}_{G}+\left(\frac{\widetilde{M}_{1}^{2} M T^{\alpha}}{\alpha \Gamma(\alpha)}\right)^{2} N_{G}\right)<1$.

Step II. We claim that $\Psi_{2}$ is compact. In [14], we have proved that $\Psi_{2}$ maps bounded sets into bounded sets of $\mathscr{F}_{b}$ and $\Psi_{2}$ maps bounded sets into equicontinuous sets of $\mathscr{B}_{b}$. It is enough to prove that $\Psi_{2}$ maps $B_{r}$ into a precompact set in $B_{r}$. Define an operator $\Psi_{2}^{\varepsilon, \delta}$ on $B_{r}$ by

$$
\begin{aligned}
\Psi_{2}^{\varepsilon, \delta} y(t)= & \alpha \int_{0}^{t-\varepsilon} \int_{\delta}^{\infty}(t-s)^{\alpha-1} L^{-1} \theta \xi_{\alpha}(\theta) S\left((t-s)^{\alpha} \theta\right) \mathrm{d} \theta f\left(s, y_{s}+\tilde{\phi}_{s}\right) \mathrm{d} s \\
& +\alpha \int_{0}^{t-\varepsilon} \int_{\delta}^{\infty}(t-s)^{\alpha-1} L^{-1} \theta \xi_{\alpha}(\theta) S\left((t-s)^{\alpha} \theta\right) \mathrm{d} \theta B u^{\lambda}(s) \mathrm{d} s \\
& +\alpha \int_{0}^{t-\varepsilon} \int_{\delta}^{\infty}(t-s)^{\alpha-1} L^{-1} \theta \xi_{\alpha}(\theta) S\left((t-s)^{\alpha} \theta\right) \mathrm{d} \theta \sigma(s) \mathrm{d} B^{H}(s) .
\end{aligned}
$$


Since $S(t), t>0$ is a compact operator, the set $\left\{\Psi_{2}^{\varepsilon, \delta} y(t), y \in B_{r}\right\}$ is precompact in $U$ for every $\varepsilon \in(0, t), \delta>0$. For each $y \in B_{r}$

$$
\begin{aligned}
& \mathrm{E}\left\|\Psi_{2}^{\varepsilon, \delta} y(t)-\Psi_{2}^{\varepsilon, \delta} y(t)\right\|^{2} \\
& \leq 6 \alpha^{2} \mathrm{E}\left\|\int_{0}^{t} \int_{0}^{\delta}(t-s)^{\alpha-1} L^{-1} \theta \xi_{\alpha}(\theta) S\left((t-s)^{\alpha} \theta\right) \mathrm{d} \theta f\left(s, y_{s}+\tilde{\phi}_{s}\right) \mathrm{d} s\right\|^{2} \\
& +6 \alpha^{2} \mathrm{E}\left\|\int_{t-\varepsilon}^{t} \int_{\delta}^{\infty}(t-s)^{\alpha-1} L^{-1} \theta \xi_{\alpha}(\theta) S\left((t-s)^{\alpha} \theta\right) \mathrm{d} \theta f\left(s, y_{s}+\tilde{\phi}_{s}\right) \mathrm{d} s\right\|^{2} \\
& +6 \alpha^{2} \mathrm{E}\left\|\int_{0}^{t} \int_{0}^{\delta}(t-s)^{\alpha-1} L^{-1} \theta \xi_{\alpha}(\theta) S\left((t-s)^{\alpha} \theta\right) \mathrm{d} \theta B u^{\lambda}(s) \mathrm{d} s\right\|^{2} \\
& +6 \alpha^{2} \mathrm{E}\left\|\int_{t-\varepsilon}^{t} \int_{\delta}^{\infty}(t-s)^{\alpha-1} L^{-1} \theta \xi_{\alpha}(\theta) S\left((t-s)^{\alpha} \theta\right) \mathrm{d} \theta B u^{\lambda}(s) \mathrm{d} s\right\|^{2} \\
& +6 \alpha^{2} \mathrm{E}\left\|\int_{0}^{t} \int_{0}^{\delta}(t-s)^{\alpha-1} L^{-1} \theta \xi_{\alpha}(\theta) S\left((t-s)^{\alpha} \theta\right) \mathrm{d} \theta \sigma(s) \mathrm{d} B^{H}(s)\right\|^{2} \\
& +6 \alpha^{2} \mathrm{E}\left\|\int_{t-\varepsilon}^{t} \int_{\delta}^{\infty}(t-s)^{\alpha-1} L^{-1} \theta \xi_{\alpha}(\theta) S\left((t-s)^{\alpha} \theta\right) \mathrm{d} \theta \sigma(s) \mathrm{d} B^{H}(s)\right\|^{2} \\
& :=6 \alpha^{2} \sum_{i=1}^{6} J_{i} .
\end{aligned}
$$

By using Hölder inequality and the assumption (B1) we have

$$
\begin{aligned}
J_{1} & \leq \widetilde{M}_{1}^{2} M^{2} \int_{0}^{t}(t-s)^{\alpha-1} \mathrm{~d} s \int_{0}^{t}(t-s)^{\alpha-1} \mathrm{E}\left\|f\left(s, y_{s}+\tilde{\phi}_{s}\right)\right\|^{2} \mathrm{~d} s\left(\int_{0}^{\delta} \theta \xi_{\alpha}(\theta) \mathrm{d} \theta\right)^{2} \\
& \leq\left(\frac{M \widetilde{M}_{1} t^{\alpha}}{\alpha}\right)^{2}\left(N_{f} r^{\prime}+k_{f}\right)\left(\int_{0}^{\delta} \theta \xi_{\alpha}(\theta) \mathrm{d} \theta\right)^{2},
\end{aligned}
$$

and

$$
\begin{aligned}
J_{2} & \leq{\widetilde{M_{1}}}^{2} M^{2} \int_{t-\varepsilon}^{t}(t-s)^{\alpha-1} \mathrm{~d} s \int_{t-\varepsilon}^{t}(t-s)^{\alpha-1} \mathrm{E}\left\|f\left(s, y_{s}+\tilde{\phi}_{s}\right)\right\|^{2} \mathrm{~d} s\left(\int_{0}^{\infty} \theta \xi_{\alpha}(\theta) \mathrm{d} \theta\right)^{2} \\
& \leq\left(\frac{M \widetilde{M}_{1} \varepsilon^{\alpha}}{\alpha}\right)^{2}\left(N_{f} r^{\prime}+k_{f}\right)\left(\int_{0}^{\infty} \theta \xi_{\alpha}(\theta) \mathrm{d} \theta\right)^{2} .
\end{aligned}
$$

Since

$$
\begin{aligned}
\mathrm{E}\left\|u^{\lambda}(s)\right\|^{2} & \leq \frac{4\left(M_{B} M \widetilde{M}_{1}\right)^{2}}{\lambda^{2} \Gamma(\alpha)^{2}}\left\{\left(M \widetilde{M_{1}} M_{1}\right)^{2} \mathrm{E}\|\phi(0)\|^{2}+2 \mathrm{E}\left\|z_{T}\right\|^{2}\right. \\
& +\left(M \widetilde{M_{1}} M_{1}\right)^{2} \widetilde{N_{G}}\|\phi\|_{s_{1}}^{2}+\widetilde{M}_{1}^{2}\left(\widetilde{N_{G}} r^{\prime}+\widetilde{k_{g}}\right) \\
& +2 c H(2 H-1) T^{2 H-1} \int_{0}^{T}\|\hat{\varphi}(s)\|_{L_{2}^{0}(W, U)}^{2} \mathrm{~d} s \\
& \left.+\mu_{A G}+\mu_{f}+c H(2 H-1)\left(\frac{M \widetilde{M}_{1}}{\Gamma(\alpha)}\right)^{2} N_{\sigma}\right\} \equiv \frac{4\left(M_{B} M \widetilde{M_{1}}\right)^{2}}{\lambda^{2} \Gamma(\alpha)^{2}} N_{u},
\end{aligned}
$$

where

$$
\mu_{A G}=2\left(\frac{M \widetilde{M_{1} T^{\alpha}}}{\alpha \Gamma(\alpha)}\right)^{2}\left(N_{G} r^{\prime}+k_{g}\right) \text { and } \mu_{f}=2\left(\frac{M \widetilde{M_{1}} T^{\alpha}}{\alpha \Gamma(\alpha)}\right)^{2}\left(N_{f} r^{\prime}+k_{f}\right) \text {, }
$$

it follows that 


$$
\begin{aligned}
J_{3} & \leq \widetilde{M}_{1}^{2} M^{2} \int_{0}^{t}(t-s)^{\alpha-1} \mathrm{~d} s \int_{0}^{t}(t-s)^{\alpha-1} \mathrm{E}\left\|B u^{\lambda}(s)\right\|^{2} \mathrm{~d} s\left(\int_{0}^{\delta} \theta \xi_{\alpha}(\theta) \mathrm{d} \theta\right)^{2} \\
& \leq\left(\frac{M \widetilde{M_{1}} M_{B} t^{\alpha}}{\alpha}\right)^{2} \frac{4\left(M_{B} M \widetilde{M_{1}}\right)^{2}}{\lambda^{2} \Gamma(\alpha)^{2}} N_{u}\left(\int_{0}^{\delta} \theta \xi_{\alpha}(\theta) \mathrm{d} \theta\right)^{2},
\end{aligned}
$$

and

$$
\begin{aligned}
J_{4} & \leq \widetilde{M}_{1}^{2} M^{2} \int_{t-\varepsilon}^{t}(t-s)^{\alpha-1} \mathrm{~d} s \int_{t-\varepsilon}^{t}(t-s)^{\alpha-1} \mathrm{E}\left\|B u^{\lambda}(s)\right\|^{2} \mathrm{~d} s\left(\int_{0}^{\infty} \theta \xi_{\alpha}(\theta) \mathrm{d} \theta\right)^{2} \\
& \leq\left(\frac{M \widetilde{M_{1}} \varepsilon^{\alpha}}{\alpha}\right)^{2} \frac{4\left(M_{B} M \widetilde{M_{1}}\right)^{2}}{\lambda^{2} \Gamma(\alpha)^{2}} N_{u}\left(\int_{0}^{\infty} \theta \xi_{\alpha}(\theta) \mathrm{d} \theta\right)^{2} .
\end{aligned}
$$

For the last parts $J_{5}, J_{6}$ when $\alpha>1-H$, we have

$$
\int_{0}^{T} \int_{0}^{T}(T-s)^{\alpha-1}(T-t)^{\alpha-1}|s-t|^{2 H-2} \mathrm{~d} s \mathrm{~d} t=\frac{B(\alpha, 2 H-1)}{2(\alpha+H-1)} T^{2(\alpha+H-1)},
$$

which imply that

$$
\begin{aligned}
J_{5}= & \mathrm{E}\left\|\sum_{n=1}^{\infty} \int_{0}^{t} \int_{0}^{\delta}(t-s)^{\alpha-1} L^{-1} \theta \xi_{\alpha}(\theta) S\left((t-s)^{\alpha} \theta\right) \mathrm{d} \theta \sigma(s) Q^{\frac{1}{2}} e_{n} \mathrm{~d} \beta_{n}^{H}(s)\right\|^{2} \\
= & \sum_{n=1}^{\infty} \mathrm{E}\left\|\int_{0}^{t} \int_{0}^{\delta}(t-s)^{\alpha-1} L^{-1} \theta \xi_{\alpha}(\theta) S\left((t-s)^{\alpha} \theta\right) \mathrm{d} \theta \sigma(s) Q^{\frac{1}{2}} e_{n} \mathrm{~d} \beta_{n}^{H}(s)\right\|^{2} \\
\leq & c H(2 H-1)\left(M \widetilde{M}_{1}\right)^{2} \int_{0}^{t} \int_{0}^{t}(t-u)^{\alpha-1}(t-v)^{\alpha-1}|u-v|^{2 H-2} \\
& \times\|\sigma(u)\|_{L_{2}^{0}(W, U)}\|\sigma(v)\|_{L_{2}^{0}(W, U)} \mathrm{d} u \mathrm{~d} v\left(\int_{0}^{\delta} \theta \xi_{\alpha}(\theta) \mathrm{d} \theta\right)^{2},
\end{aligned}
$$

and

$$
\begin{aligned}
J_{6}= & \mathrm{E}\left\|\sum_{n=1}^{\infty} \int_{t-\varepsilon}^{t} \int_{\delta}^{\infty}(t-s)^{\alpha-1} L^{-1} \theta \xi_{\alpha}(\theta) S\left((t-s)^{\alpha} \theta\right) \mathrm{d} \theta \sigma(s) Q^{\frac{1}{2}} e_{n} \mathrm{~d} \beta_{n}^{H}(s)\right\|^{2} \\
= & \sum_{n=1}^{\infty} \mathrm{E}\left\|\int_{t-\varepsilon}^{t} \int_{\delta}^{\infty}(t-s)^{\alpha-1} L^{-1} \theta \xi_{\alpha}(\theta) S\left((t-s)^{\alpha} \theta\right) \mathrm{d} \theta \sigma(s) Q^{\frac{1}{2}} e_{n} \mathrm{~d} \beta_{n}^{H}(s)\right\|^{2} \\
\leq & c H(2 H-1)\left(M \widetilde{M}_{1}\right)^{2} \int_{t-\varepsilon}^{t} \int_{t-\varepsilon}^{t}(t-u)^{\alpha-1}(t-v)^{\alpha-1}|u-v|^{2 H-2} \\
& \times\|\sigma(u)\|_{L_{2}^{0}(W, U)}\|\sigma(v)\|_{L_{2}^{0}(W, U)} \mathrm{d} u \mathrm{~d} v\left(\int_{0}^{\infty} \theta \xi_{\alpha}(\theta) \mathrm{d} \theta\right)^{2} .
\end{aligned}
$$

Then, for each $y \in B_{r}$,

$$
\mathrm{E}\left\|\Psi_{2}^{\varepsilon, \delta} y(t)-\Psi_{2}^{\varepsilon, \delta} y(t)\right\|^{2} \rightarrow 0 \text { as } \varepsilon \rightarrow 0, \delta \rightarrow 0 .
$$

Therefore, there are relatively compact sets arbitrary close to the set $\left\{\Psi_{2} y(t), y \in B_{r}\right\}$ is precompact in $B_{r}$. By Arzela-Ascoli's theorem, $\Psi_{2}$ is compact. By Sadakovskii's fixed point theorem (Theorem 3.1), the operator $\Psi$ has a fixed point which is a solution to the system (1.1).

Theorem 3.3 Assume that the conditions of Theorem 3.2 and $\left(\mathbb{H}_{0}\right)$ hold. In addition, the functions $f$ is uniformly bounded on its domain. Then, the fractional control system (1.1) is approximately controllable on $[0, T]$. 
Proof. Let $x^{\lambda}$ be a fixed point of the operator $\Phi^{\lambda}$. Using the stochastic Fubini theorem, we can get

$$
\begin{aligned}
x^{\lambda}(T)= & z_{T}-\lambda\left(\lambda I+\Gamma_{0}^{T}\right)^{-1}\left[-\mathscr{T}_{L}(T)(L \phi(0)-G(0, \phi))\right. \\
& \left.-L^{-1} G\left(t, x_{t}\right)+E z_{T}+\int_{0}^{T} \hat{\varphi}(s) \mathrm{d} B^{H}(s)\right] \\
& +\lambda \int_{0}^{T}\left(\lambda I+\Gamma_{s}^{T}\right)^{-1}(T-s)^{\alpha-1} L^{-1} A \mathscr{S}_{L}(T-s) G\left(s, x_{s}\right) \mathrm{d} s \\
& +\lambda \int_{0}^{T}\left(\lambda I+\Gamma_{s}^{T}\right)^{-1}(T-s)^{\alpha-1} \mathscr{S}_{L}(T-s) f\left(s, x_{s}^{\lambda}\right) \mathrm{d} s \\
& +\lambda \int_{0}^{T}\left(\lambda I+\Gamma_{s}^{T}\right)^{-1}(T-s)^{\alpha-1} \mathscr{S}_{L}(T-s) \sigma(s) \mathrm{d} B^{H}(s) .
\end{aligned}
$$

It follows from the property of $f, G$ that there exists $C>0$ such that $\left\|f\left(s, x_{s}^{\lambda}\right)\right\|^{2} \leq C$ and $\left\|A G\left(s, x_{s}^{\lambda}\right)\right\|^{2} \leq C$. Then there is a subsequence denoted by $\left\{f\left(s, x_{s}^{\lambda}\right), A G\left(s, x_{s}^{\lambda}\right)\right\}$ weakly converging to $\{f(s), G\}$. Thus, from the above equation, we obtain

$$
\begin{aligned}
& \mathrm{E}\left\|x^{\lambda}(T)-z_{T}\right\|^{2} \\
& \leq 8\left\|\lambda\left(\lambda I+\Gamma_{0}^{T}\right)^{-1}\left[\mathrm{E}_{T}-\mathscr{T}_{L}(T)(L \phi(0)-G(0, \phi))\right]\right\|^{2} \\
& +8 \mathrm{E}\left\|\lambda\left(\lambda I+\Gamma_{0}^{T}\right)^{-1}\left[L^{-1} G\left(t, x_{t}\right)\right]\right\|^{2} \\
& +8 c H(2 H-1) T^{2 H-1} \mathrm{E} \int_{0}^{T}\left\|\lambda\left(\lambda I+\Gamma_{0}^{T}\right)^{-1} \hat{\varphi}(s)\right\|_{L_{2}^{0}(W, U)}^{2} \mathrm{~d} s \\
& +8 \mathrm{E}\left(\int_{0}^{T}(T-s)^{\alpha-1}\left\|\lambda\left(\lambda I+\Gamma_{s}^{T}\right)^{-1} L^{-1} A \mathscr{S}_{L}(T-s)\left[G\left(s, x_{s}^{\lambda}\right)-G(s)\right]\right\| \mathrm{d} s\right)^{2} \\
& +8 \mathrm{E}\left(\int_{0}^{T}(T-s)^{\alpha-1}\left\|\lambda\left(\lambda I+\Gamma_{s}^{T}\right)^{-1} L^{-1} A \mathscr{S}_{L}(T-s) G(s)\right\| \mathrm{d} s\right)^{2} \\
& +8 \mathrm{E}\left(\int_{0}^{T}(T-s)^{\alpha-1}\left\|\lambda\left(\lambda I+\Gamma_{s}^{T}\right)^{-1} \mathscr{S}_{L}(T-s)\left[f\left(s, x_{s}^{\lambda}\right)-f(s)\right]\right\| \mathrm{d} s\right)^{2} \\
& +8 \mathrm{E}\left(\int_{0}^{T}(T-s)^{\alpha-1}\left\|\lambda\left(\lambda I+\Gamma_{s}^{T}\right)^{-1} \mathscr{S}_{L}(T-s) f(s)\right\| \mathrm{d} s\right)^{2} \\
& +8 c H(2 H-1) \int_{0}^{T} \int_{0}^{T}(T-s)^{\alpha-1}(T-t)^{\alpha-1}|s-t|^{2 H-2} \\
& +\left\|\lambda\left(\lambda I+\Gamma_{s}^{T}\right)^{-1} \mathscr{S}_{L}(T-s) \sigma(s)\right\|_{L_{2}^{0}(W, U)}^{2}\left\|\lambda\left(\lambda I+\Gamma_{t}^{T}\right)^{-1} \mathscr{S}_{L}(T-t) \sigma(t)\right\|_{L_{2}^{0}(W, U)}^{2} \mathrm{~d} s \mathrm{~d} t .
\end{aligned}
$$

On the other hand, by assumption $\left(\mathbb{H}_{0}\right)$ for all $0 \leq s \leq T$, the operator $\lambda\left(\lambda I+\Gamma_{s}^{T}\right)^{-1} \rightarrow 0$ strongly as $\lambda \rightarrow 0+$, and moreover $\left\|\lambda\left(\lambda I+\Gamma_{s}^{T}\right)^{-1}\right\|<1$. Thus, by the Lebesgue dominated convergence theorem and the compactness of $\mathscr{S}_{L}(t)$, we can get $\mathrm{E}\left\|x^{\lambda}(T)-z_{T}\right\|^{2} \rightarrow 0$ as $\lambda \rightarrow 0+$. This gives the approximate controllability of (1.1).

\section{Conclusion}

We consider the following Sobolev-type fractional neutral stochastic differential 
equations driven by fractional Brownian motion with infinite delay:

$$
\begin{cases}{ }^{c} D_{t}^{\alpha}\left[L x(t)-G\left(t, x_{t}\right)\right]=A x(t)+f\left(t, x_{t}\right)+B u(t)+\sigma(t) \frac{\mathrm{d}}{\mathrm{d} t} B^{H}(t), & t \in(0, T], \\ x(t)=\phi(t), & t \in(-\infty, 0] .\end{cases}
$$

where $u(\cdot)$ is a control function. Inspired by [14], we show the existence of solution and approximate controllability of (1.1).

\section{Funding}

The Project-sponsored by NSFC (No. 11571071).

\section{References}

[1] Mahmudov, N.I. (2003) Approximate Controllability of Semilinear Deterministic and Stochastic Evolution Equations in Abstract Spaces. SIAM Journal on Control and Optimization, 42, 1604-1622. https://doi.org/10.1137/S0363012901391688

[2] Mahmudov, N.I. (2008) Approximate Controllability of Evolution Systems with Nonlinear Analysis: Theory, Methods \& Applications, 68, 536-546. https://doi.org/10.1016/j.na.2006.11.018

[3] Sakthivel, R., Ganesh, R. and Suganya, S. (2012) Approximate Controllability of Fractional Neutral Stochastic System with Infinite Delay. Reports on Mathematical Physics, 70, 291-311. https://doi.org/10.1016/S0034-4877(12)60047-0

[4] Fu, X. and Mei, K. (2009) Approximate Controllability of Semilinear Partial Functional Differential Systems. Journal of Dynamical and Control Systems, 15, 425-443. https://doi.org/10.1007/s10883-009-9068-x

[5] Sakthivel, R., Ren, Y. and Mahmudov, N.I. (2011) On the Approximate Controllability of Semilinear Fractional Differential Systems. Computers \& Mathematics with Applications, 62, 1451-1459. https://doi.org/10.1016/j.camwa.2011.04.040

[6] Baleanu, D. (2012) Fractional Calculus: Models and Numerical Methods. World Scientific, Boston. https://doi.org/10.1142/8180

[7] Kilbas, A.A., Srivastava, H.M. and Trujillo, J.J. (2006) Theory and Applications of Fractional Differential Equations. Elsevier, Amsterdam.

[8] Gurtin, M.E. and Pipkin, A.C. (1968) A General Theory of Heat Conduction with Finite Wave Speed. Archive for Rational Mechanics and Analysis, 31, 113-126. https://doi.org/10.1007/BF00281373

[9] Nunziato, J.W. (1971) On Heat Conduction in Materials with Memory Q. Appl. Math., 29, 187-204.

[10] Revathi, P., Sakthivel, R. and Ren, Y. (2016) Stochastic Functional Differential Equations of Sobolev-Type with Infinite Delay. Statistics \& Probability Letters, 109, 68-77. https://doi.org/10.1016/j.spl.2015.10.019

[11] Wang, J., Feckan, M. and Zhou, Y. (2014) Controllability of Sobolev Type Fractional Evolution Systems. Dynamics of Partial Differential Equations, 11, 71-87. https://doi.org/10.4310/DPDE.2014.v11.n1.a4

[12] Biagini, F. and Hu, Y. (2008) Øksendal B., Zhang T.: Stochastic Calculus for Fractional Brownian Motion and Applications. Springer, New York. https://doi.org/10.1007/978-1-84628-797-8

[13] Mishura, Y.S. (2008) Stochastic Calculus for Fractional Brownian Motion and Related Processes. Springer, Berlin. https://doi.org/10.1007/978-3-540-75873-0 
[14] Han, J. and Yan, L. (2018) Controllability of a Stochastic Functional Differential Equation Driven by a Fractional Brownian Motion. Advances in Difference Equations, 2018, 104. https://doi.org/10.1186/s13662-018-1565-3

[15] Feckan, M., Wang, J. and Zhou, Y. (2013) Controllability of Fractional Functional Evolution Equations of Sobolev Type via Characteristic Solution Operators. Journal of Optimization Theory and Applications, 156, 79-95. https://doi.org/10.1007/s10957-012-0174-7

[16] Cui, J. and Yan, L. (2011) Existence Result for Fractional Neutral Stochastic Integro-Differential Equations with Infinite Delay. Journal of Physics A: Mathematical and Theoretical, 44, 335201. https://doi.org/10.1088/1751-8113/44/33/335201

[17] Mahmudov, N.I. and Denker, A. (2000) On Controllability of Linear Stochastic Systems. International Journal of Control, 73, 144-151.

https://doi.org/10.1080/002071700219849

[18] Guendouzi, T. and Idrissi, S. (2012) Approximate Controllability of Fractional Stochastic Functional Evolution Equations Driven by a Fractional Brownian Motion. ROMAI Journal, 8, 103-117.

[19] Daher, S.J. (1978) On a Fixed Point Principle of Sadovskii. Nonlinear Analysis: Theory, Methods \& Applications, 2, 643-645.

https://doi.org/10.1016/0362-546X(78)90012-3

[20] Li, Y. and Liu, B. (2007) Existence of Solution of Nonlinear Neutral Stochastic Differential Inclusions with Infinite Delay. Stochastic Analysis and Applications, 25, 397-415. https://doi.org/10.1080/07362990601139610 